\title{
Evolution of probability measures by cellular automata on algebraic topological Markov chains
}

\author{
ALEJANDRO MAASS and SERVET MARTÍNEZ
}

Departamento de Ingeniería Matemática and Centro de Modelamiento Matemático Departamento de Ingeniería Matemática, UMR 2071 UCHILE-CNRS, Universidad de Chile, Casilla 170-3, Correo 3, Santiago, Chile

\begin{abstract}
In this paper we review some recent results on the evolution of probability measures under cellular automata acting on a fullshift. In particular we discuss the crucial role of the attractiveness of maximal measures. We enlarge the context of the results of a previous study of topological Markov chains that are Abelian groups; the shift map is an automorphism of this group. This is carried out by studying the dynamics of Markov measures by a particular additive cellular automata.

Many of these topics were within the focus of Francisco Varela's mathematical interests.
\end{abstract}

Key terms: Topological Markov chains, cellular automata, maximal measure, entropy, additive cellular automata.

\section{INTRODUCTION}

Let $A$ be a finite set of symbols and $M=\left(M_{a, b}\right.$ $: a, b \in A)$ be a $\{0,1\}$-valued matrix indexed by the alphabet $A$. We consider the set of allowed two-sided sequences of symbols in $A$ or configurations, $X_{M}=\left\{x=\left(x_{l}\right)_{i \in} z \cap i \in \mathbf{Z}\right.$, $\left.M_{x i x i+1}=1\right\}$

The matrix $M$ specifies a set of constraints of the configurations, $M_{a, b=0}$ means that the path from $a$ to $b$ is forbidden, and on the contrary, when $M_{a, b=1}$ it is allowed. In the event that all entries of $M$ are equal to one, the set $X_{M}$, is called fullshift and denoted by $A^{\mathrm{z}}$.

A cellular automaton (CA) defined on $X_{M}$ is a map $F: X_{M} \rightarrow X_{M}$ such that for $x \in X_{M}$ and $i \in \mathrm{Z},(F x)_{i}=f\left(x_{i-r} \ldots x_{i+r}\right)$ where $r \in \mathbf{N}$ is the radius and $f: A^{2 r+1} \rightarrow A$ is a given local rule (see Figure 1). When the local rule does not depend on left coordinates $\chi_{i-r}, \ldots, \chi_{i-i}$, we say it is a one-sided CA. In this case the local rule is defined by $f: A^{r+1} \rightarrow A$ and $(F x)_{i}=f\left(x_{i-r} \ldots x_{i+r}\right)$.

Among CA we distinguish the shift map $\sigma_{M}: X_{M} \rightarrow X_{M}$ such that $\left(\sigma_{M} x\right)_{i}=x_{i+1}$. This map codes time in the sense that $x_{i}$ represents the state of system at time $i$. Observe that $\sigma_{\mathrm{M}}$ is one-sided, and it is induced by the local rule with $r=1, f: A^{2} " A, f\left(x_{0} x_{1}\right)=x_{1}$.. It is evident that $F^{\circ} \sigma_{M}=\sigma_{M}{ }^{o} F$ for any CA $F ; X_{M} \rightarrow X_{M}$. When $X_{M}$ is a fullshift, we use the notation $\sigma_{\mathrm{A}}$ instead of $\sigma_{M}$.

Cellular automata have served as models of systems which present self organization, and the study of their dynamics has been the object of several papers over the last three decades. One approach to the study of such behavior is to consider a 'natural' probability measure $\mu$ of full support in $X_{M}$ (which represents a realistic amount of initial conditions) and the sequence of iterated measures $\left(F^{n} \mu: n \in N\right)$, where $\left(F^{n} \mu\right)(B)=$ $\mu\left(F^{n}(B)\right)$ for every measurable set $B$. The behavior of such a sequence has been studied in particular contexts in recent years: (Lind, 1984; Mass and Martínez, 1998; Ferrari et al, 2000; Pivato and Yassawir, 2001, 2002). One of the primary objectives of this study is to link the limit behavior of this sequence with probability measures of maximal entropy, in other words, the way in which the CA goes to

1991 Mathematics Subject Classification. Primary: 54H20; Secondary: 37B20. 
a maximal excited state in equilibrium.

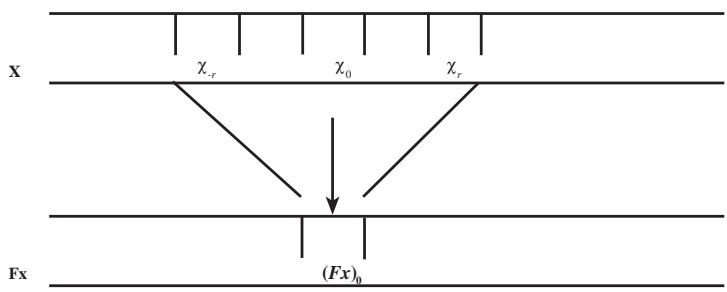

FIGURE 1: CA given by $\mathrm{F}$

It is easy to verify that the above iteration admits several limit behaviors. To illustrate, consider in the fullshift $\{0,1\}^{\mathrm{Z}}$ the mod $2 \mathrm{CA}$ given by $(F x)_{i}=x_{i}+x_{i+1}$, where addition is modulo 2. Then: $\left(F^{2 n} x\right)_{i}=x_{i}+x_{i+2}$, and

$$
\left(F^{2^{n}-1} x\right)_{i}=\sum_{j=0}^{2^{n}-1} x_{i+j},
$$

so for any Bernoulli measure $\mu$ the limits of $\left(F^{n} \mu: n \in N\right)$ are different along these subsequences.

In order to study the equilibrium, a natural point of view is to consider the convergence of the temporal mean

$$
\frac{1}{N} \sum_{n=0}^{N-1} F^{n} \mu
$$

for a class of translation invariant probability measures $\mu$ on $X_{M}$. In fact, any limit for this sequence is also invariant for the $\mathrm{CA}$. The main existing results concern fullshifts. This study began with Lind's pioneer (1984) work for the mod $2 \mathrm{CA}$ and initial Bernoulli measures, where it is proven that the temporal mean converges to the uniform Bernoulli measure, which is the maximal entropy measure for $F$ and $\sigma_{\{0,1\}}$. The same result was shown for larger classes of group cellular automata and more general initial probability measures in (Mass and Martínez, 1998; Ferrari et al, 2000).

Ferrari et al (2000) used regeneration theory of stochastic processes with finite state space. Lind's point of view is that of harmonic analysis: the cellular automaton is viewed as an endomorphism of the product group and the uniform Bernoulli measure is its Haar measure. This approach was followed in Pivato and Yassawir (2001 and 2002), where the authors proved the same kind of results for the class of probability measures they called harmonically mixing, and the family of additive cellular automata verifying a diffusive condition. The previous results motivated us to study those sensitive cellular automata which can be decomposed as the product of an additive cellular automaton with one having an equi-continuous direction (Host et al, 2002).

Let us provide some notation and background.

\section{ALPHABET, WORDS, SEQUENCES}

A finite set of symbols $A$ is called alphabet, and by $A$ we mean the set of finite sequences or words $w=w_{0} \ldots w_{n-1}$ with letters in $A$ and $|w|$ denotes the length of $w \in A^{+}$. Given $x=$ $\left(x_{j}\right)_{j \in A} \in A^{Z}$ and $i \leq j$ in $\mathrm{Z}$, or $x=x_{0} \ldots x_{n} \in A^{+}$and $i \leq j$ in $\{0, \ldots, n\}$, we denote by $x[i, j]=x_{i} \ldots x_{j}$ the finite word in $x$ between coordinates $i$ and $j$.

Let $X_{M}=\left\{x=\left(x_{j}\right)_{j \in A} \in A^{Z}: \cap j \in A E, M_{x j, x j+l}=1\right\}$ for $M$ a $\{0,1\}$-valued matrix indexed by $A$ This is the topological Markov chain induced by $M$.

For any $n \in \mathrm{N}$ we define the set of words of length $n$ in $X_{M}$ by $L_{n}\left(X_{M}\right)=\left\{w \in A^{*}: \exists x \in X_{M}\right.$, $x[0, n-1]=w\}$

The set of all words or language of $X_{M}$ is $L\left(X_{M}\right)=\cup_{n \in l} L_{n}\left(X_{M}\right)$. Given $w \in L\left(X_{M}\right)$ and $i \in \mathrm{N}$, the cylinder set starting in coordinate $i$ with word $w$ is $[w]_{i}=\left\{x \in X_{M}: x[i, i+|w|-1]=w\right\}$.

Cylinder sets are a fundamental base for the topology of $X_{M}$, which is metrizable and compact.

Let $F: X_{M} \rightarrow X_{M}$ be a CA. We can apply its local rule $f$ (of radius $r$ ) to words. Given $w=$ $w_{0} \ldots w_{n} \in L_{n}\left(X_{M}\right)$ with $|w| \geq 2 r+1$, then $f(w)$ $=f\left(w_{0} \ldots w_{2 r}\right) f\left(w_{1} \ldots w_{2 r+1}\right) f\left(w_{n-2 r} \ldots w_{n}\right)$.

This allows us to define by induction the $N$ th power of $f, f^{N}: L_{2 N r+1}\left(X_{M}\right) \rightarrow L_{l}\left(X_{M}\right)$, by $f^{N}$ $\left(w_{-N r} \ldots w_{N r}\right)=f^{N-1}\left(f\left(w_{-N r} \ldots w_{-N r+r}\right) \ldots f\left(w_{N r-r} \ldots w_{N r}\right)\right)$.

\section{CLASSES OF CA}

A CA $F: X_{M} \rightarrow X_{M}$ is said to be right permutative if for every $w_{n} \in L_{r 2}\left(X_{M}\right)$ the map $f(w,$.$) defined by f(w,).(\mathrm{a})=f(w \mathrm{a})$, when $w_{n}$ $w a \in L_{r 2+1}\left(X_{M}\right)$, is one-to-one. Analogously we define left permutative and bipermutative CA.

Assume $\left(X_{M},+\right)$ is a group. We say that $F$ is additive if for every $x, y \in X L_{M} F(x+y)=F$ $(x)+F(y)$. By Kitchens' results (1987), any 
topological Markov chain that is a group and such that the shift $\sigma_{M}$ is an automorphism, is equivalent to one induced by a group $\left(L_{1}\right.$ $\left.\left(X_{M}\right),+\right)$ in such a way that $x+y=\left(x_{i}+y_{i}\right)_{i \in Z}$. Therefore the local rule of an additive CA $F$ satisfies $f(w+w)=f(w)+f\left(w^{\prime}\right)$ for $w, w^{\prime} \in L_{r 2+1}$ $\left(X_{M}\right)$. A particular class of CA additive is that whose local rules $f$ can be written $f\left(w_{0} w_{1}\right)=$ $w_{0}+w_{1}$ for every $w_{0} w_{1} \in L_{2}\left(X_{M}\right)$, which is equivalent to $F=x+\sigma_{\mathrm{M}(\mathrm{x})}$ These $\mathrm{CA}$ are right permutative.

Another class of right permutative $\mathrm{CA}$ are additive-translation CA. They are defined on a product space by $F$ × $G: X_{M} \times X_{M^{\prime}} \rightarrow X_{M} \times X_{M^{\prime}}$, where $\left(X_{M},+\right)$ is an Abelian group, $F: X_{M} \rightarrow$ $X_{M}$ is additive, and $G: X_{M^{\prime}} \rightarrow X_{M}$ is defined by $(G x)=g\left(x_{l+1}\right)$ with $g: L_{1}\left(X_{M^{\prime}}\right) \rightarrow\left(X_{M^{\prime}}\right)$ a oneto-one local rule. This class of CA appear naturally as the representation of right permutative CA whose local rules verify some algebraic conditions in [Host et al, 2002].

\section{Probabilistic background}

Relevant shift invariant probability measures are Bernoulli and Markov measures. Let us describe them in $A^{z}$. It is enough to give its value in the set of cylinder sets.

Let $\pi=\left(\pi_{a}: a \in A\right)$ be a probability vector; the Bernoulli measure $\mu$ is given by $\mu_{\pi}\left(\left[a_{0}, \ldots, a_{n}\right]_{i}\right)=\prod_{j=0}^{n} \pi_{a_{j}}$. Under this measure all coordinates are independent, so there is no memory.

Markov measures have memory one, and they are defined by a stochastic matrix $P$ and its stationary probability vector $\pi$ and we denote it by $\mu$ It is given by

$\mu_{\pi_{i} P}\left(\left[a_{0}, \ldots, P, a_{n}\right]_{i}\right)=\pi_{a_{0}} P_{a_{0}, a_{1}} \ldots P_{a_{n-1}, a_{n}}$

This measure is supported by $X_{M}^{a_{n-1}, a_{n}} \subseteq A^{z}$. where $M_{a, b}=1$ if and only if $P_{a, b}>0$. Observe that if $P_{a, b}=\pi_{b}$ for any $a, b \in A$ then $\mu_{\pi, P}=\mu_{n}$.

Let us introduce a relevant class of infinite memory probability measures. Denote $\mathrm{N}^{*}=$ $\mathrm{N} \backslash\{0\}$ and $-\mathrm{N}^{*}=\left\{-i: i \in \mathrm{N}^{*}\right\}$. Let $\mu$ be a shift invariant probability measure on $A^{Z}$ For $\underline{w} \in$ $A^{- \text {I.* }^{*}}$ let $\mu_{w}$ be the conditional measure on $A^{\mathrm{N}}$ given $\underline{w}$ that is for any $m \geq 0$ and $a_{0}, \ldots, a_{m} \in A$, $\mu_{w}\left\{x_{0}=a_{0}, \ldots, x_{m}=a_{m}\right\}=\mu\left\{x_{0}=a_{0}, \ldots, x_{m}=a_{m} \mid x_{i}=w_{i}, i<0\right\}$

The measure $\mu$ is said to have complete connections if it satisfies

$$
\forall a_{0}, \ldots, a_{m} \in A, \forall \underline{w} \in A^{-\mathbf{i}^{*}}, \mu_{\underline{w}}\left\{x_{0}=a_{0}, \ldots, x_{m}=a_{m}\right\}>0 .
$$

In this case define for $m \geq 0$

$$
\gamma_{m}=\sup \left\{\left|\frac{\mu_{\underline{\underline{w}}}\left\{x_{0}=a\right\}}{\mu_{\underline{\underline{v}}}\left\{x_{0}=a\right\}}-1\right|: a \in A, \underline{\underline{v}}, \underline{\underline{w}} \in A^{-\mathbf{i}{ }^{*}}, v_{i}=w_{i}, i \in[-m,-1]\right\} .
$$

The measure $\mu$ has summable decay if $\sum_{m=0}^{\infty} \gamma_{m}<\infty$. This is a uniform continuity condition on $\mu_{\underline{w}}$ as a function of $\underline{W}$

\section{Entropy}

One of the fundamental invariants in ergodic theory is entropy, both topological and measure-theoretical. Here we introduce them in the context of cellular automata. Let $F: X_{M}$ $\rightarrow X_{M}$ be a CA and $\mu$ a $F$-invariant probability measure defined on measurable sets of $X_{M}$, that is, $\mu(C)=\mu\left(F^{-1}(C)\right)$ for every cylinder set $C$ Let $\alpha_{n}=\left\{[w]_{-n}: w \in L_{2 n+1}\left(X_{M}\right)\right\}$ be the partition of centered cylinders of length $2 n+$ 1 in $X_{M}$. The dynamics of this partition $\alpha_{n}$ under the transformation $F$ is given by the sequence of partitions $\left(\alpha_{n}(F, N) N \in \mathrm{I}\right)$, where $\alpha_{n}(F, N)=\left\{B_{0} \cap F^{-1} B_{1} \cap \ldots \cap F^{-N+1} B_{N-1}: B_{j} \in \alpha_{n}, j \in\{0, \ldots, N-1\}\right\}$.

The entropy of $F$ with respect to $\mu$ is

$$
h_{\mu}(F)=\lim _{n \rightarrow \infty} \lim \frac{1}{N} \sum_{B \in \alpha_{n}(F, N)}(-\mu(B) \log \mu(B)) \text {. }
$$

If $F \sigma_{M}$ it holds that

$$
h_{\mu}\left(\sigma_{M}\right)={ }_{n \rightarrow \infty} \frac{1}{n} \sum_{B \in \alpha_{n}}(-\mu(B) \log \mu(B)) \text {. }
$$

There is a topological counterpart. The topological entropy of $F$ is

$$
h_{\text {top }}(F)=\lim _{n \rightarrow \infty} \lim _{N \rightarrow \infty} \frac{1}{N} \#\left\{B \in \alpha_{n}(F, N): B \neq 0\right\} \text {. }
$$

The variational principle links both notions of entropy in the following way:

$h_{\text {top }}(F)=\sup \left\{h_{\mu}(F): \mu\right.$ is a $\mathrm{F}$-invariant probability measure $\}$.

A probability measure $\mu$ satisfying $h_{\text {top }}(F)$ $=h_{\mu}(F)$ is said to be of maximal entropy. These measures carry the maximal amount of information of the system.

Maximal entropy measures for topological Markov chains $X_{M}$ such that $M^{m}>0$ for some $m \in$ Í can be explicitly described in terms of algebraic characteristics of the matrix. Let $u, v$ be left and right eigenvectors of $M$ associated to the eigenvalue $\lambda$ of maximum modulus, that is, $u M=\lambda u, M v=\lambda v$. By Perron- 
Frobenius theorem $\lambda>0$ and also $u>0, v>0$. Assume $\sum_{a \in A} u_{a} v_{a}=1$. Define the stochastic matrix $P_{M}$ by

$$
\left(P_{M}\right)_{a, b}=\frac{v_{b}}{\lambda v_{a}} M_{a, b}, \quad a, b \in A .
$$

The probability vector $\pi_{M}=\left(\left(\pi_{M}\right)_{\mathrm{a}}: a \in A\right)$ given by $\left(\pi_{M}\right)_{\mathrm{a}}=u_{a} v_{a}$ is the stationary vector for this matrix, that is, $\pi_{M} \mathrm{P}_{M}=\pi_{M}$. Then the associated stationary Markov measure $\mu_{\pi_{M}, P_{M}}$ supported in $X_{M}$ verifies

$\mu_{\pi_{M}, P_{M}}\left(\left[a_{0} \ldots a_{n-1}\right]_{\mathrm{i}}\right)=\lambda^{-n} u_{a_{0}} v_{a_{n-1}} M_{a_{0}, a_{1}} \ldots M_{a_{n-2}, a_{n-1}}$.

If $X_{M}$ is the fullshift $A^{Æ}$, the maximal measure is the uniform Bernoulli measure $\lambda_{A}^{\mathbb{E}}$ such that

$$
\lambda_{A}^{\mathbb{E}}\left(\left[a_{0} \ldots a_{n-1}\right]_{\mathrm{i}}\right)=(\# A)^{-n} .
$$

Temporal mean of iteration of measures by $C A$

Let $F: X_{M} \rightarrow X_{M}$ be a CA and $\mu$ be a shift invariant probability measure on $X_{M}$. As usual, $F^{n} \mu$ denotes the measure given by $\left(F^{n}\right.$ $\mu(\mathrm{B})=\mu\left(F^{-n} \mu\right)$ for $B$ a measurable set. We will discuss the convergence in distribution of the temporal mean measure

$$
M_{\mu}^{N}(F)=\frac{1}{N} \sum_{n=0}^{N-1} F^{n} \mu .
$$

If this limit exists as $\mathrm{N} \rightarrow \infty$, we denote it by $M \mu(F)$.

CONVERGENCE OF TEMPORAL MEANS TO MAXIMAL ENTROPY MEASURE

Let $F: X_{M} \rightarrow X_{M}$ be a CA. In this section we will supply conditions ensuring the convergence of $M_{\mu}^{N}(F)$ to a maximal entropy measure for $\sigma_{M}$. This is shown for different kinds of translation invariant probability measures $\mu$. In particular we will consider the case of stationary Markov probability measures on $X_{M}$.

\section{Convergence of temporal means for additive} $C A$

Let $(K,+)$ be a finite Abelian group and $F$ : $K^{Z} \rightarrow K^{Z}$ be an additive CA. In a recent article, Pivato and Yassawir (2002) used a harmonic analysis framework to study the convergence of $M_{\mu}^{N}(F)$ to the uniform Bernoulli measure of $\mathrm{K}^{2}$. For this purpose they introduce the notion of harmonically mixing measures. A shift invariant probability measure $\mu$ on $\mathrm{K}^{2}$ is called harmonically mixing if for all $\varepsilon>=$, there is some $m>0$ so that for every character $\chi \in \hat{K}^{\mathrm{Z}}$ with rank $(\chi)>m$ it holds $\left|\int_{K^{2}} \chi d \mu\right|<\varepsilon$, where rank $(\chi)$ is the number of coordinates of dependence of $\chi$.

The statement (2.1) $\lim _{N \rightarrow \infty} M_{\mu}^{N}(F)=\lambda_{K}^{F} \quad$ was shown to hold for any additive $\mathrm{CA}$ and any harmonically mixing measure in Pivato and Yassawir (2001) (a previous proof for $K=Z_{p}, p$ a prime number, was shown in Pivato and Yassawir (2002)). The proof is based on the fact that for every non-trivial character $\chi \in \hat{K}^{\mathrm{z}}$, there is some subset $D_{\chi} \subset \mathbf{I}$ of density one, so that rank $\left(\chi^{o} F^{j}\right)$ goes to $\infty$ when $j$ tends to $\infty$ inside $D_{X}$. Moreover, Pivato and Yassawir (2002) also proved that Bernoulli measures and Markov measures whose transition matrices have strictly positive entries are harmonically mixing.

Our approach to (2.1) was probabilistic. In Ferrari et al (2000) we show that (2.1) holds for any measure with complete connections and summable decay memory for groups where $\# K$ is a power of a prime number. In addition, in Host et al (2002) we extend the result to any group by showing that measures with complete connections and summable decay are harmonically mixing.

In addition to these results, in an ergodic context, when $\# K=p$ a prime number, if all weak limits of $\left(\mathrm{M}_{\mu}^{N}(F): N \in \mathbf{I}\right)$ are ergodic for the shift map and have positive entropy for $F$, we prove in Host et al (2002) that (2.1) holds.

Convergence of temporal means for additive-translation $C A$

In Host et al (2002) we consider more general classes of CA, those which admit a product representation as an additive-translation CA. They contain one-sided right permutative $\mathrm{CA}$ of radius 1 acting on a fullshift such that the local rules $f: A^{2} \rightarrow A$ are associative, that is, there is a one-to-one map $\psi: A \rightarrow A$ such that $\psi:[f(f(a, b), c)]=f(a, f(a, c))$; or satisfy a $N$ scaling condition, that is,

$$
\left.f^{N}\left(x_{0}, \ldots, x_{N}\right)\right]=f\left(x_{0}, x_{N}\right)
$$


where $f^{N}$ is the $N$-th power of $f$. Therefore temporal means can be computed for classes of CA that a priori do not have any algebraic structure. A main point in this decomposition is that in one coordinate we have a group structure and in the other a translation CA for which temporal means are periodic.

Let $F \times G:(K \times A)^{E} \rightarrow(K \times A)^{\mathrm{Z}}$ be an additive-translation $\mathrm{CA}$ and let $\mu$ be a shift invariant probability measure on $(K \times A)^{\mathrm{z}}$. Consider a weak limit $\tilde{\mu}$ of $\left(M_{\mu}^{N}(F \times G): N \in \mathbf{I}\right)$. This measure has two natural projections in each coordinate, $\pi_{K} \tilde{\mu}$ and $\pi_{A} \tilde{\mu}$, but a priori it is not true that $\tilde{\mu}=\pi_{K} \tilde{\mu} \times \pi_{A} \tilde{\mu}$. We prove in Host et al, (2002) that if $\pi_{K} \tilde{\mu}=\lambda_{K}^{\mathrm{z}}$ and for some $L \in Æ$ the CA $G \circ \sigma_{A}^{L}$ has zero entropy for $\pi_{A} \tilde{\mu}$, then $\tilde{\mu}=\lambda_{K}^{\mathrm{Z}} \times \pi_{A} \tilde{\mu}$.

A corollary of the previous result and the properties described in previous subsection is: for any shift invariant probability measure $\mu$ with complete connections and summable decay it holds

$$
M_{\mu}(F \times G)=\lambda_{K}^{E} \times v,
$$

where $v=\mathrm{M}_{\pi_{A} \mu}(G)$. In fact, $G \circ \sigma_{A}^{-1}$ has zero entropy because it is a $\mathrm{CA}$ of radius $\mathrm{O}$.

\section{Convergence of temporal means for a particular additive $C A$}

In this section we announce a new result on convergence of temporal means to the maximal entropy measure, in the context of additive CA acting on an Abelian group $\left(X_{M},+\right)$. We will not give a proof of the result but we will illustrate it on a particular example constructed on $\left(X_{M},+\right)$ given in Kitchens (1987).

In the example, the alphabet is $A=\oiint_{4} \times Z_{2}$ $=\{0,1,2,3\} \times\{0,1\}$, which is an Abelian group with coordinate operations addition $\bmod 4$ and addition mod 2 respectively (that we call + in the sequel for simplicity). The matrix $M$ verifies $M_{\left(a_{1}, a_{2}\right),\left(b_{1}, b_{2}\right)}=1$ if and only if $b_{2}$ $=a_{2}+a_{2} \bmod 2$. It can be verified that $\left(X_{M}\right.$, $+)$ is a group and $\sigma_{M}: X_{M} \rightarrow X_{M}$ is an automorphism of $\left(X_{M},+\right)$. In fact $\mathrm{M}_{\left(a_{1}+a_{1}^{\prime}, a_{2}+a_{2}^{\prime}\right),\left(b_{1}+b_{1}^{\prime}, b_{2}+b_{2}^{\prime}\right)}=1$ whenever $b_{2}=a_{2}+a_{2}$ $\bmod 2$ and $b_{2}=a_{1}^{\prime}+a_{2}^{\prime} \bmod 2$. Finally we consider the additive CA $F: X_{M} \rightarrow X_{M}$, given by $F:(x)=x+\sigma_{M}(x)$ (it is called group CA in Ferrari et al, $(2000,2000))$. Below we denote $\sigma_{E}=\sigma_{2}$ and $\sigma_{E}=\sigma_{4}$.

Let $\mu_{M}$ be the measure of maximal entropy for $\sigma_{M}$ defined in Section 2. It is the Markovian measure defined by the transition matrix

$$
P_{M}=\left[\begin{array}{cccccccc}
1 / 4 & 0 & 1 / 4 & 0 & 1 / 4 & 0 & 1 / 4 & 0 \\
0 & 1 / 4 & 0 & 1 / 4 & 0 & 1 / 4 & 0 & 1 / 4 \\
1 / 4 & 0 & 1 / 4 & 0 & 1 / 4 & 0 & 1 / 4 & 0 \\
0 & 1 / 4 & 0 & 1 / 4 & 0 & 1 / 4 & 0 & 1 / 4 \\
1 / 4 & 0 & 1 / 4 & 0 & 1 / 4 & 0 & 1 / 4 & 0 \\
0 & 1 / 4 & 0 & 1 / 4 & 0 & 1 / 4 & 0 & 1 / 4 \\
1 / 4 & 0 & 1 / 4 & 0 & 1 / 4 & 0 & 1 / 4 & 0 \\
0 & 1 / 4 & 0 & 1 / 4 & 0 & 1 / 4 & 0 & 1 / 4
\end{array}\right]
$$

and its stationary vector is

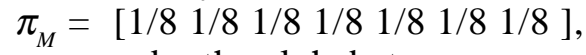

where we order the alphabet as

$\{(0,0),(0,1),(1,0),(1,1),(2,0),(2,1),(3,0),(3,1)\}$

Consider another shift invariant Markov probability measure $\mu_{\pi, P}$ defined by a stochastic matrix $P$ and its stationary probability vector $\pi$ full supported by $X_{M}$, that is, $M_{a, b}>0$ if and only if $P_{a, b}>0$.

\section{Theorem 1}

We have $\mathrm{M}_{\mu_{\pi, p}}(F)=\mu_{M}$.

Proof. The elements of $X_{M}$ are denoted by $z=(x, y)$. We set $G: A_{4}^{E} \rightarrow A_{4}^{E}$ to be the CA induced by $F$ on $\boldsymbol{F}_{4}^{\mathbf{E}}$. It can be proven inductively that

$$
F^{n} z=\sum_{i=0}^{n}\left(\begin{array}{l}
n \\
i
\end{array}\right) \sigma_{M}^{i} z=z+\sum_{i=1}^{n}\left(\begin{array}{l}
n \\
i
\end{array}\right)\left(\sigma_{4}^{i} x, \sigma_{4}^{i-1} x \bmod 2\right)
$$

Since $F$ is right permutative it can be shown that for any allowed word $a_{0}, \ldots, a_{m-1} \in L_{m}\left(X_{M}\right)$ and $n \geq 1$ there are two words $b_{0} \ldots b_{m}, c_{0} \ldots c_{\mathrm{m}}$ $\in \mathrm{Z}^{*}{ }_{4}$ such that the following equality of sets holds

$$
\begin{aligned}
& \left\{z \in X_{M}: F^{n} z[0, m-1]=a_{0} \ldots a_{m-1}\right\}= \\
& \left\{z \in X_{M}: G^{n-1} x[0, m]=b_{0} \ldots b_{m}\right\} \cup\left\{z \in X_{M}: G^{n-1} x[0, m]=c_{0} \ldots c_{m}\right\},
\end{aligned}
$$

the union being disjoint.

We compute

$$
\mu_{\pi, P}\left(\left\{z \in X_{M}: G^{n} x[0, m]=b_{0} \ldots b_{m}\right\}\right)
$$

and

$$
\mu_{\pi, P}\left(\left\{z \in X_{M}: G^{n} x[0, m]=c_{0} \ldots c_{m}\right\}\right)
$$

for $n \geq 0$. Since $\mu_{\pi, P}$ is Markovian, the same arguments used in the fundamental construction of Ferrari et al (2000) allow us to prove 
$\lim _{N \rightarrow \infty} \frac{1}{N} \sum_{n=0}^{N-1} \mu_{\pi, P}\left(\left\{z \in X_{M}: G^{n} x[0, m]=b_{0} \ldots b_{m}\right\}\right)=\left(\frac{1}{4}\right)^{m+1}$

and

$\lim _{N \rightarrow \infty} \frac{1}{N} \sum_{n=0}^{N-1} \mu_{\pi, P}\left(\left\{z \in X_{M}: G^{n} x[0, m]=c_{0} \ldots c_{m}\right\}\right)=\left(\frac{1}{4}\right)^{m+1}$

Therefore

$\lim _{N \rightarrow \infty} \frac{1}{N} \sum_{n=0}^{N-1} \mu_{\pi, P}\left(\left\{z \in X_{M}: F^{n} z[0, m-1]=a_{0} \ldots a_{m-1}\right\}\right)$

$=\frac{1}{8}\left(\frac{1}{4}\right)^{m-1}=\mu_{M}\left(\left[a_{0} \ldots a_{m-1}\right]_{0}\right)$.

This shows the result.

\section{ACKNOWLEDGMENTS}

The authors acknowledge financial support from Millennium Nucleus Information and Randomness ICM P01-005.

\section{REFERENCES}

FERRARI P, MAASS A, MARTÍNEZ S, NEY P (2000) Cesàro mean distribution of group automata starting from measures with summable decay. Ergodic Theory and Dynamical Systems 20: 1657-1670

KITCHENS B (1987) Expansive dynamics on zerodimensional groups. Ergodic Theory and Dynamical Systems 7: 249-261

LIND D (1984) Applications of ergodic theory and sofic systems to cellular automata. Physica D 10: 36-44

MAASS A, MARTÍNEZ S (1998) On Cesàro limit distribution of a class of permutative cellular automata. Jrnl of Statist Phys 90: 435-452

PIVATO M, YASSAWI R (2002) Limit Measures for Affine Cellular Automata, Ergodic Theory and Dynamical Systems 22: 1269-1287 